\title{
Habilidades discursivas en estudiantes universitarios. Un estudio exploratorio con alumnos de Contabilidad y Administración de la Universidad de Sonora
}

(Discursive skills in college students. An exploratory study with students of Accounting and Administration at University of Sonora)

\author{
María Edith Araoz Robles iD , Universidad de Sonora, Sonora, México \\ ANa Bertha de la VARA EstradA ID Universidad de Sonora, Sonora, México \\ Alma Liliana GaRCÍA CAÑEdo ${ }^{\text {iD }}$, Universidad de Sonora, Sonora, México
}

Volumen 1, Número 1

Edición especial. Febrero de 2016

p. $84-99$

Este número se publicó el 5 de febrero de 2016

Artículo recibido: 9 de marzo de 2015

Artículo aprobado: 4 de noviembre de 2015

ISSN: 2448-5942, doi: https://doi.org/10.36799/el.v1i1.23

Citar este artículo como:

Araoz Robles, M. E., De La Vara Estrada, A. B., y García Cañedo, A. L. (2016). Habilidades discursivas en estudiantes universitarios. Un estudio exploratorio con alumnos de Contabilidad y Administración de la Universidad de Sonora. Estudios גambda. Teoría y práctica de la didáctica en lengua y literatura, 1(1), 84-99. https://doi.org/10.36799/el.v1i1.23

Derechos de autor: El autor o autores conservan en todo momento sus derechos morales y patrimoniales sobre la obra; la obra no se puede alterar, transformar o ampliar; siempre debe reconocerse la autoría del documento

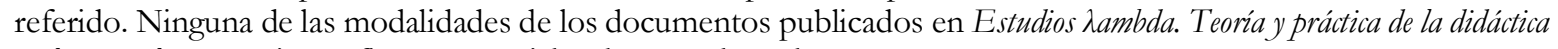
en lengua y literatura tienen fines comerciales de naturaleza alguna.

Los contenidos de este artículo están bajo la licencia de Creative Commons Atribución No Comercial- Sin Derivadas 4.0 Internacional @ $\odot \Theta \Theta$ 


\title{
Habilidades discursivas en estudiantes universitarios. Un estudio exploratorio con alumnos de Contabilidad y Administración de la Universidad de Sonora
}

\author{
(Discursive skills in college students. An exploratory study with students of Accounting \\ and Administration at University of Sonora)
}

\author{
MARÍA EDITH ARAOZ ROBLES ${ }^{1}$ \\ ANA BERTHA DE LA VARA ESTRADA ${ }^{2}$ \\ ALMA LILIANA GARCía CAÑEDO ${ }^{3}$
}

\begin{abstract}
RESUMEN
Este trabajo forma parte de un proyecto interdisciplinario realizado por docentes de la Academia en Lengua Escrita del Departamento de Letras y Lingüística y del Departamento de Contabilidad. El objetivo general es examinar las habilidades de lectura y escritura de los alumnos inscritos en las licenciaturas de Contaduría Pública, Administración, Informática Administrativa y Mercadotecnia. El interés por realizar un proyecto conjunto surge ante el hecho concreto de observar dificultades en las habilidades de lectura y escritura de estudiantes de estos programas educativos. Nuestra premisa principal es que la lectura y la escritura son herramientas fundamentales en el proceso de aprendizaje, toda vez que es a través de ellas que adquirimos, construimos y reconstruimos el conocimiento. El proyecto se planificó en cuatro etapas: 1) Construcción de instrumentos para explorar las habilidades de lectura y escritura: una encuesta, un examen diagnóstico y obtención de la muestra representativa. 2) Aplicación de los instrumentos a la muestra seleccionada. 3) Revisión y evaluación de resultados. 4) Propuestas de solución. A la fecha, hemos concluido las tres primeras etapas de la investigación y el diagnóstico muestra que las habilidades comunicativas de los estudiantes evaluados son insuficientes y preocupantes, sobre todo si las consideramos en el contexto universitario. Presentamos aquí los resultados obtenidos así como algunas propuestas y estrategias de solución a la problemática encontrada.
\end{abstract}

PALABRAS CLAVE: Lectura, escritura, aprendizaje, proyecto interdisciplinario

\begin{abstract}
This paper presents the first part of an interdisciplinary project realized by instructors of the Written Language Academy in both the Department of Literature and Linguistics and the Accounting Department from University of Sonora. The overall goal is to examine the reading and writing abilities of undergraduate students in the Departments of Accounting, Administration, Administrative Information, and Marketing. Our main concern starts from a group project observation in order to identify difficulties in reading and writing skills of students while taking these programs. Our premise is that reading and writing are essential tools in the learning process, since it is through them that we acquire, build, and rebuild knowledge. The project was planned in four stages: 1) Building of tools to explore reading and writing skills: a survey, a diagnosis test and the obtainment of results from a student sample. 2) Application of the tools to the representative sample. 3) Reviewing and evaluation of results. 4) Proposals for solutions. Up to now we have arrived to the conclusion that the first three stages of the investigation and the diagnosis shows that the evaluated students' communication skills are not yet good enough and that it is something that must be taken care of soon, above all considering their academic context. We also present here the results obtained and some proposals and strategies to solve problems found.
\end{abstract}

KEYWORDS: Reading, writing, learning, interdisciplinary project

\footnotetext{
${ }^{1}$ Maestra de tiempo completo del Departamento de Letras y Lingüística de la Universidad de Sonora. Candidata a doctor en Ciencias Sociales. Líneas de investigación: comprensión lectora y producción textual. earaoz@.capomo.uson.mx

2 Maestra de tiempo completo del Departamento de Letras y Lingüística de la Universidad de Sonora. Maestría en Literatura Hispanoamericana. Líneas de investigación: comprensión lectora y producción textual. bdvara@capomo.uson.mx

${ }^{3}$ Maestra de tiempo completo del Departamento de Contabilidad de la Universidad de Sonora.
}

Artículo recibido: 9 de marzo de 2015

Artículo enviado a corrección: 25 de septiembre de 2015

Aprobado: 4 de noviembre de 2015

Araoz, De la Varay García doi: https://doi.org/10.36799/el.v1i1.23 Volumen 1, Número 1, Año 2016, ISSN: 2448-5942 
...porque leer y escribir forman parte del quehacer profesional/académico de los graduados que esperamos formar y porque elaborar y comprender escritos son los medios ineludibles para aprender los contenidos conceptuales de las disciplinas que estos graduados también deben conocer.

Paula Carlino

\section{INTRODUCCIÓN}

La lectura y la escritura son las principales herramientas en el proceso de enseñanza-aprendizaje, es a través de ellas que adquirimos, construimos y reconstruimos el conocimiento; no obstante, en ocasiones se convierten en las principales dificultades que enfrentan los estudiantes que ingresan a la educación superior, dificultades que son inherentes al aprendizaje de nuevos conocimientos. ¿Qué debemos hacer los docentes y las instituciones educativas ante este hecho? Coincidimos con Castro Azuara en que "la tarea de las instituciones de nivel superior es facilitar a los estudiantes su ingreso a estas nuevas culturas de lo escrito" (2013, 14), luego entonces, nuestra tarea como docentes es iniciarlos en el conocimiento de las prácticas discursivas especializadas y facilitarles así el desarrollo de habilidades para investigar, seleccionar la información, reflexionar sobre ésta, evaluarla y profundizar en su conocimiento. Estas habilidades les posibilitarán no sólo acercarse al conocimiento especializado, sino también, como menciona Cassany (2006), serán instrumentos mediante los cuales construirán su identidad personal, académica y profesional.

En el caso de los estudiantes de la Universidad de Sonora, la problemática sobre las habilidades comunicativas es similar a la que se presenta en otras instituciones de educación superior (Carlino 2012; Castro 2013; Martínez 2005; Parodi 2005 y Sánchez 2013). Como docentes, ${ }^{4}$ advertimos dicha problemática y decidimos realizar un estudio exploratorio para investigar su magnitud. Este trabajo tiene el propósito de presentar los resultados obtenidos en una investigación diagnóstica interdisciplinaria, realizada por docentes del Departamento de Contabilidad (ECA) y de la Academia en Lengua Escrita del Departamento de Letras y Lingüística.

\footnotetext{
${ }^{4}$ Las coautoras de este trabajo impartimos, desde nuestras áreas disciplinares, diferentes asignaturas a estudiantes del
} Departamento de Contabilidad.

Araoz, De la Varay García doi: https://doi.org/10.36799/el.v1i1.23 Volumen 1, Número 1, Año 2016, ISSN: 2448-5942 
Los objetivos de la investigación diagnóstica consistieron en explorar las habilidades de lectura y escritura de estudiantes y, con base en los resultados, como un primer paso, enunciar y promover propuestas de solución a la problemática encontrada. Nuestros sujetos de estudio fueron estudiantes del Departamento de Contabilidad de la Universidad de Sonora, inscritos en las licenciaturas de Contaduría, Administración, Informática Administrativa y Mercadotecnia.

\section{MARCO TEÓRICO CONCEPTUAL}

Partimos de la concepción de lectura como un proceso cognitivo mediante el cual adquirimos, construimos y reconstruimos el conocimiento. Leer es una práctica discursiva que implica un antes, un durante y un después, en donde, de manera individual, tenemos la oportunidad de plantearnos preguntas, de pensar, inferir, decidir qué es importante y qué no lo es (Araoz, Guerrero, Galindo, Villaseñor y De la Vara 2010, 42). Al leer ponemos en práctica operaciones de pensamiento que nos permiten comprender un texto, lo cual significa poder interpretarlo y poder hacer una representación mental de sus significados. La comprensión de un texto implica identificar tanto su temática como la idea eje que el autor quiere comunicarnos; significa reconocer cuál fue su propósito al escribir el texto y distinguir la estructura que empleó, entre muchos otros aspectos. En el proceso de comprensión se da una transacción entre la información escrita, nuestros conocimientos previos y el contexto, ${ }^{5}$ es esta transacción autorlector-contexto la que hace posible comprender lo que leemos.

Existe "una relación estrecha entre la comprensión lectora y el desarrollo intelectual, de tal manera que leer bien es la mejor forma de aprender a escribir bien” (Araoz et al., 2010, 71). Aprender a escribir bien significa saber organizar ideas, construir textos con coherencia, adaptar el estilo según el destinatario, según el tema a tratar y de acuerdo con el tipo de texto que queremos redactar. En este sentido, Carlino afirma que al escribir se ponen en práctica procesos de aprendizaje que no siempre ocurren si no hay producción escrita, "no hay apropiación de ideas sin reelaboración” $(2012,24)$, toda vez que escribir es un proceso que nos_permite comprender, pensar, integrar y transformar el conocimiento. Al escribir, organizamos nuestro

\footnotetext{
${ }^{5}$ En un sentido amplio, retomando algunas nociones de Calsamiglia y Tusón (2007), entendemos por contexto todas aquellas situaciones de comunicación, sean lingüísticas, socioculturales o intertextuales, que intervienen para que un texto pueda ser interpretado, para que las palabras y enunciados adquieran su significado preciso.
}

Araoz, De la V aray García doi: https://doi.org/10.36799/el.v1i1.23 Volumen 1, Número 1, Año 2016, ISSN: $2448-5942$ 
pensamiento, y estimulamos el análisis crítico. Además, dado que la escritura tiene mayor grado de formalidad que el habla, la escritura de un texto, sobre todo de un texto académico, nos obliga a hacer uso de los aspectos normativos de la lengua para poder expresarnos con claridad y precisión. ${ }^{6}$

La lectura y la escritura son prácticas discursivas que se desarrollan en un entorno social y mediante las cuales no sólo se transmite información, sino que también se construye. Asimismo, como señala Cassany, al leer y escribir, "se crean las identidades del autor y del lector de los textos." $(2008,79)$.

\section{METODOLOGÍA}

Al realizar la investigación sobre habilidades de lectura y escritura en estudiantes universitarios, se utilizó una metodología que proporcionó datos cuantitativos y, a partir de éstos, se realizó el análisis de la problemática. Para ello, se construyó un instrumento mediante el cual se exploraron las habilidades de lectoescritura que poseen los estudiantes de la ECA, se emitió un diagnóstico y se analizaron alternativas y propuestas de solución.

\section{- Elaboración del instrumento}

Para la construcción del instrumento exploratorio se tomó en cuenta nuestra experiencia docente con los estudiantes del Departamento de Contabilidad. ${ }^{7}$

Partimos de la premisa de que en el proceso de comprensión lectora intervienen múltiples factores, como el cognitivo, el afectivo, el social y el ambiental; por ello, no es factible hablar de un procedimiento específico que permita evaluar dicho proceso. Sin embargo, es posible tomar en cuenta algunos aspectos importantes mediante los cuales logramos reconocer el nivel de comprensión: podemos decir que el estudiante comprendió un texto cuando puede explicarlo,

\footnotetext{
6 Aspectos normativos como la acentuación, la puntuación y la ortografía son fundamentales para lograr una comunicación escrita eficiente. Estos elementos nos permiten organizar las ideas de manera coherente y darle sentido al texto.

7 Cabe mencionar que un instrumento de características similares se elaboró en 2012 y fue avalado por los miembros de la Academia de Literatura del Departamento de Letras y Lingǘstica; este examen se ha aplicado desde entonces a la fecha a los estudiantes de nuevo ingreso a la licenciatura en Literaturas Hispánicas. De igual manera, y a solicitud de la Maestría en Innovación Educativa, en 2011 se elaboró y aplicó un examen diagnóstico para evaluar las habilidades de lectura y escritura de los aspirantes.
} 
parafrasearlo, resumirlo, esquematizarlo, aplicarlo a otro contexto o adaptarlo a su realidad; cuando puede hablar de su importancia o cuestionarla; cuando hace conexiones con otras ideas y hechos; cuando puede predecir acciones, etcétera.

Con base en lo anterior, el examen diagnóstico se estructuró en dos partes: en la primera se registran aspectos básicos de ortografía (separación silábica, acentuación y uso adecuado de grafías), sinonimia, antinomia y puntuación; y en la segunda, se incluyen aspectos de organización y jerarquización de ideas (estructura sintáctica y semántica de la oración, esquematización) así como actividades de comprensión lectora y escritura. Ambas partes arrojaron datos mediante los cuales fue posible explorar y diagnosticar aquellos aspectos que representan mayor dificultad para los estudiantes.

- Datos sobre la obtención de la muestra.

Para la obtención de la muestra se consultaron datos de la Dirección de Servicios Escolares de la Universidad de Sonora. De acuerdo con ésta, el total de alumnos del Departamento de Contabilidad inscritos en el semestre 2012-2, era de 2988, y con base en el número de créditos aprobados, la población estudiantil se divide en tres categorías:

\begin{tabular}{|c|c|c|c|c|c|c|c|c|c|c|}
\hline & \multicolumn{3}{|c|}{ 1a. Categoría } & \multicolumn{3}{|c|}{ 2a. Categoría } & \multicolumn{3}{|c|}{ 3a. Categoría } & \\
\hline & \multicolumn{9}{|c|}{ Créditos } & \\
\hline & $0-50$ & $51-100$ & $101-150$ & $151-200$ & $201-250$ & $251-300$ & $301-350$ & $351-400$ & $401-450$ & \\
\hline Programa & \multicolumn{9}{|c|}{ Número de alumnos } & Total \\
\hline Contador Público & 150 & 197 & 101 & 172 & 102 & 119 & 125 & 81 & 13 & 1060 \\
\hline Lic. en Administración & 182 & 234 & 108 & 186 & 109 & 122 & 118 & 28 & & 1087 \\
\hline \multicolumn{11}{|l|}{ Lic. en Informática } \\
\hline Administrativa & 34 & 69 & 33 & 61 & 46 & 26 & 5 & & & 274 \\
\hline Lic. en Mercadotecnia & 118 & 124 & 42 & 101 & 49 & 117 & 16 & & & 567 \\
\hline Total General & 484 & 624 & 284 & 520 & 306 & 384 & 264 & 109 & 13 & \\
\hline Total por Categoría & \multicolumn{3}{|c|}{1392} & \multicolumn{3}{|c|}{1210} & \multicolumn{3}{|c|}{386} & 2988 \\
\hline
\end{tabular}

Tabla 1. Alumnos activos en el Periodo 2012-2, del Departamento de Contabilidad de la Unidad Regional Centro, por Créditos Aprobados 
La primera categoría, de 0 a 150 créditos, corresponde a estudiantes que cursan los primeros tres semestres de las carreras; la segunda, de 151 a 300 créditos, comprende a quienes cursan el $4^{\circ}, 5^{\circ}$ y $6^{\circ}$ semestre; y la tercera, de 301 a 450 créditos, incluye a estudiantes del $7^{\circ}, 8^{\circ}$ y $9^{\circ}$ semestre.

Previo a la aplicación del instrumento de evaluación se aplicó una muestra piloto de 10 para determinar el error que se tendría al aplicar el instrumento en su totalidad, lo que arrojó un error estándar o desviación estándar del 5.3\%, y por consiguiente un Nivel de Confianza del 94.7\% . La fórmula estadística para la obtención de la muestra es la utilizada para universo de datos finitos. (Inzunza 2012 y Munch 2009):

$\mathrm{Z}^{2} \mathrm{~N}$ pq $(1.62)^{2}(2988)(0.938)(0.062)$

$\mathrm{n}=$ - - 53 datos

$\mathrm{e}^{2} \mathrm{~N}+\mathrm{Z}^{2} \mathrm{pq}(.053)^{2}(2988)+(1.62)^{2}(0.938)(0.062)$

Donde:

$\mathrm{n}=$ número de datos representativos

Z= Nivel de confianza 94.7\%, que corresponde a 1.62 desviaciones estándar

$\mathrm{N}=$ número de alumnos

$\mathrm{p}=$ probabilidad a favor $93.8 \%$

$\mathrm{q}=$ probabilidad en contra $6.2 \%$

$\mathrm{e}=$ error estándar $5.3 \%$

- Aplicación del instrumento

Una vez obtenida la muestra de la población total se hizo una primera estratificación de acuerdo a la proporción de estudiantes de cada licenciatura, es decir, se transportó la proporción al número de encuestas que se aplicarían y se obtuvieron los siguientes resultados: 


\begin{tabular}{|l|c|c|c|}
\hline \multicolumn{1}{|c|}{ Licenciatura } & Total alumnos & $\%$ & Encuestas \\
\hline Contador Público & 1060 & 35 & 18 \\
\hline Lic. en Administración & 1087 & 37 & 20 \\
\hline Lic. en Informática Administrativa & 274 & 9 & 5 \\
\hline Lic. en Mercadotecnia & 567 & 19 & 10 \\
\hline Total & 2988 & 100 & 53 \\
\hline
\end{tabular}

Tabla 2. Primera estratificación: porcentaje de encuestas por licenciatura.

Posteriormente, se realizó una segunda estratificación para distribuir el número de encuestas por licenciatura, de acuerdo a la proporción de alumnos inscritos en cada Categoría. La distribución quedó de la siguiente manera:

\begin{tabular}{|c|c|c|c|c|}
\hline Carrera & Totales & $1^{\mathrm{a}}$ Categoría & $2^{\text {a }}$ Categoría & $3^{\mathrm{a}}$ Categoría \\
\hline \multirow{3}{*}{ C.P. } & $1060100 \%$ estudiantes & 448 & 393 & 219 \\
\hline & $\%$ & 42 & 37 & 21 \\
\hline & $18100 \%$ encuestas & 8 & 7 & 3 \\
\hline \multirow{3}{*}{ L.A. } & $1087100 \%$ estudiantes & 524 & 417 & 219 \\
\hline & $\%$ & 48 & 38 & 14 \\
\hline & $20100 \%$ encuestas & 10 & 8 & 2 \\
\hline \multirow{3}{*}{ L.I.A. } & $274100 \%$ estudiantes & 136 & 133 & 5 \\
\hline & $\%$ & 50 & 48 & 2 \\
\hline & $5100 \%$ encuestas & 2 & 2 & 1 \\
\hline \multirow{3}{*}{ L.M. } & $567100 \%$ estudiantes & 284 & 267 & 16 \\
\hline & $\%$ & 50 & 47 & 3 \\
\hline & $10100 \%$ encuestas & 5 & 4 & 1 \\
\hline
\end{tabular}

Tabla 2. Segunda estratificación: porcentaje de encuestas por categoría. 
A partir de las estratificaciones, se procedió a la aplicación del instrumento en el número y categoría correspondientes a cada carrera. Se citó a los estudiantes de acuerdo a su categoría. El tiempo destinado para la realización fue de dos horas. Veamos los resultados cuantitativos obtenidos.

\section{ANÁLISIS DE RESULTADOS}

- Resultados generales

En el Departamento de Contabilidad los resultados generales que se obtuvieron tanto en los aspectos normativos como en la organización y comprensión de ideas fueron negativos: 54.93 en una escala de 100, lo cual indica una calificación reprobatoria. En la primera parte, correspondiente a los aspectos normativos de la legua, se obtuvo una puntuación de 35.21 de 50 puntos posibles, y en la segunda, correspondiente a organización de ideas y comprensión lectora se obtuvo 19.72 de 50. En una escala de 100, esto es equivalente a calificaciones de 70.42 y 39.44 , respectivamente.

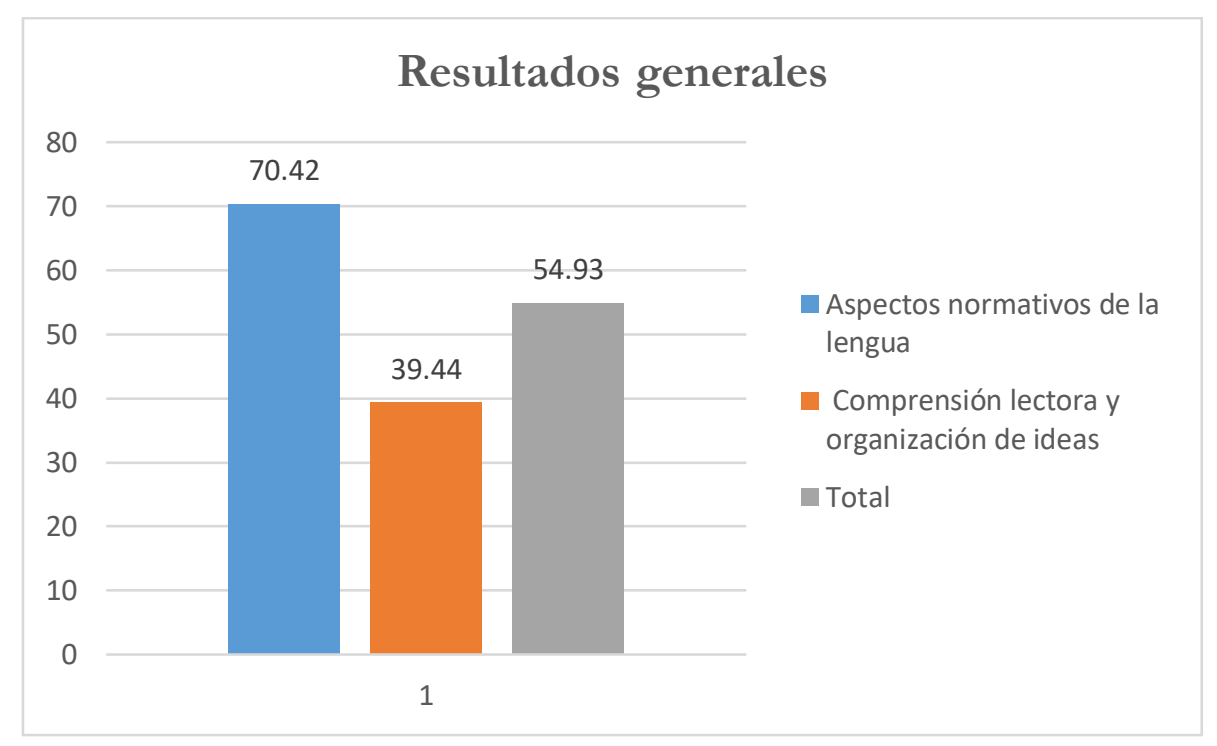

Tabla 4. Resultados cuantitativos generales del Departamento de Contabilidad. 
De estos resultados, el más alto, 63.43, fue de la licenciatura en Mercadotecnia y el más bajo, con 49.11, de la licenciatura en Informática Administrativa:

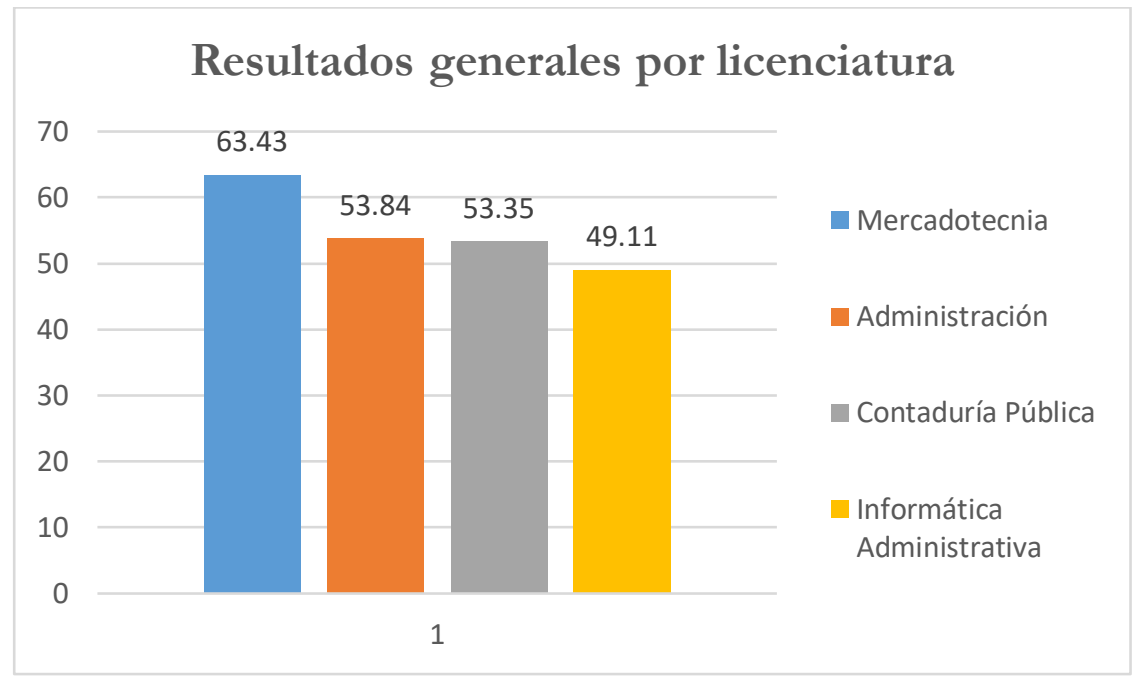

Tabla 5. Resultados generales por licenciatura.

- Resultados de aspectos normativos de la lengua por licenciatura

En esta primera parte del diagnóstico, el área de Mercadotecnia obtuvo la mayor puntuación: 77.46, mientras que Informática Administrativa obtuvo la menor con 61.02.

Como se observa en la tabla 6 , todas las licenciaturas obtuvieron resultados aprobatorios:

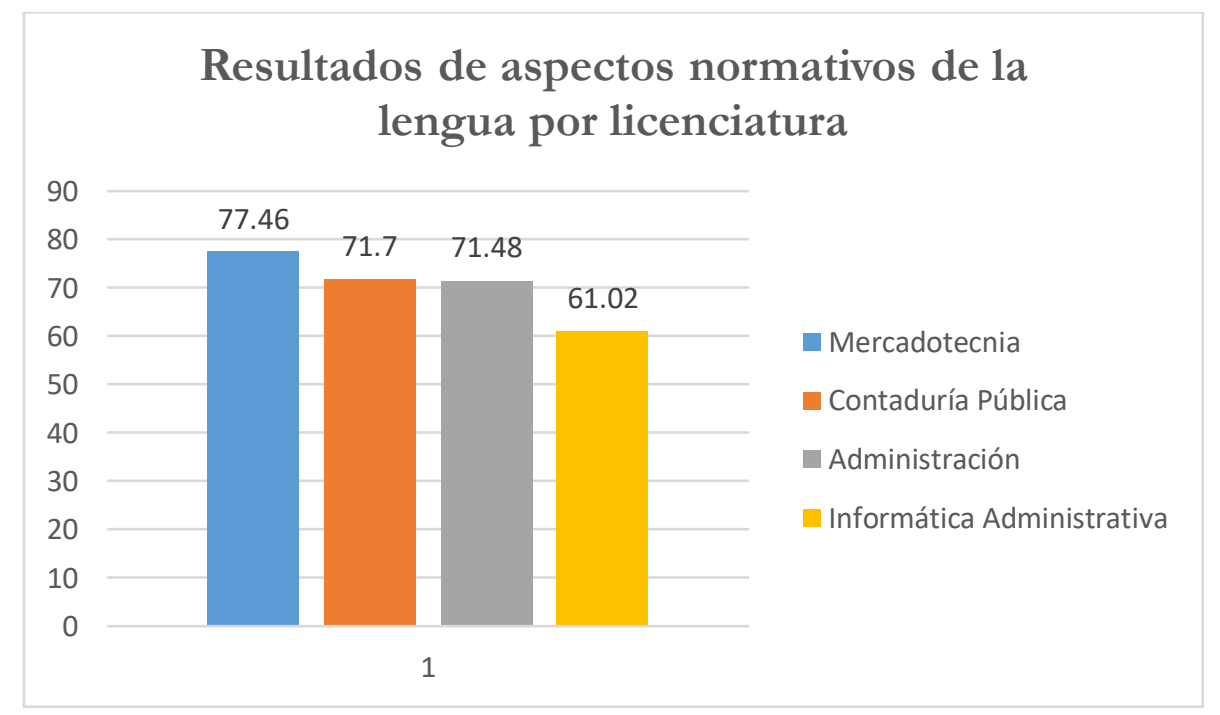

Tabla 6. Resultados obtenidos por cada licenciatura en aspectos normativos de la lengua. 
- Resultados de comprensión lectora y organización de ideas por licenciatura

Con respecto a la segunda parte del instrumento de evaluación, los resultados fueron contrastantes con respecto a los de la primera, ya que las cuatro licenciaturas presentaron resultados negativos: la que obtuvo mayor puntuación fue Mercadotecnia con 49.4, y la menor fue Contaduría Pública, con 35:

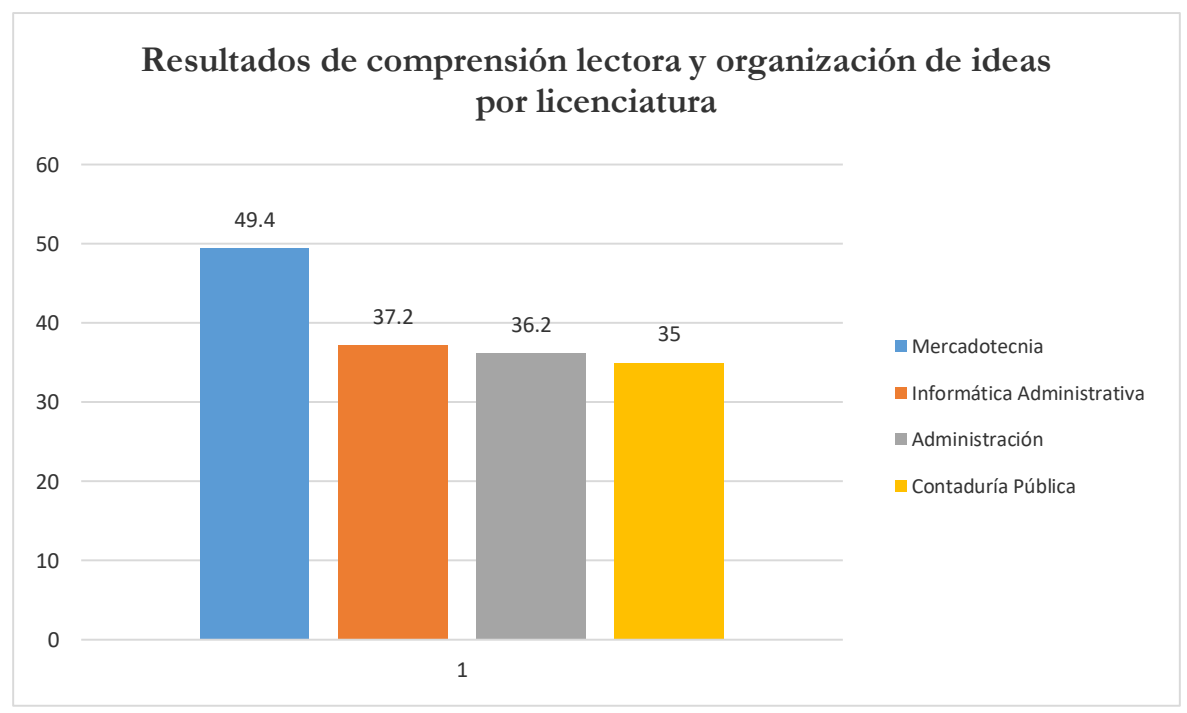

Tabla 7. Resultados obtenidos por licenciatura en aspectos de redacción y organización de ideas.

- Resultados obtenidos por los estudiantes

Aquí, los rangos se distanciaron de manera considerable: el mayor puntaje obtenido fue de 90, de un estudiante de Mercadotecnia; el más bajo fue de 26.56 de un estudiante de Contabilidad. En los aspectos normativos, fueron estudiantes de Contabilidad los que obtuvieron la máxima y la mínima puntuación en una escala de 50: 45.69 y 4.56 puntos, lo cual en escala de 100 equivale a calificaciones de 91.38 y 9.12. En comprensión lectora y organización de ideas, también en escala de 50, la máxima puntuación fue de 46 puntos de un estudiante de Mercadotecnia, la más baja fue de 2 puntos, de un estudiante de Administración. En escala de 100, estos puntajes representan calificaciones de 92 y 4, respectivamente. 
- Resultados globales por licenciatura

Los resultados globales de cada licenciatura en orden descendente son:

- Mercadotecnia fue la única del Departamento que "aprobó" la evaluación, ya que obtuvo un promedio general de 63.43. En los aspectos normativos la calificación fue de 77.46 y en comprensión de lectura y organización de ideas, de 49.4. De los estudiantes evaluados, el 60\% obtuvo resultados positivos y el 40\% negativos. La máxima calificación fue de 90 y la mínima de 42.9.

- Administración. El promedio general de esta licenciatura fue de 53.84, lo cual, en la escala de calificaciones de la Universidad de Sonora, indica que está por debajo del límite aprobatorio. En aspectos normativos obtuvo 71.48, y en comprensión y organización de ideas 36.2. De los estudiantes participantes, el 40\% aprobó y el 60\% reprobó. La máxima calificación fue de 76.86 y la menor, de 28.07 .

- Contaduría Pública. El promedio acumulado por los estudiantes de esta licenciatura fue de 53.35, lo que indica resultados negativos en este estudio exploratorio. El promedio en aspectos normativos fue de 71.7 y en redacción de 35. De los participantes, el 38.89\% aprobó y el $61.11 \%$, reprobó. La mayor calificación fue de 77.69 y la menor, de 26.56.

- Informática Administrativa. El puntaje general que obtuvo esta disciplina fue de 49.11, lo que significa resultados negativos. En los aspectos normativos alcanzaron $61.02 \%$ y en redacción y organización de ideas, 37.2\%. La mayor calificación fue de 56.25 y la menor de 38.1.

Veamos ahora el análisis de estos datos.

\section{ANÁLISIS DE DATOS}

Si partimos de la premisa de que "leer y escribir es algo tan diseminado e importante en la vida humana que afecta a todas las esferas, disciplinas y categorías" (Cassany 2008, 25), podemos diagnosticar, con base en los resultados cuantitativos expuestos, que existe una problemática preocupante en torno a las habilidades de lectura y escritura de los estudiantes de la ECA.

Aun cuando en la primera parte del examen (aspectos normativos de la lengua) obtuvieron puntajes positivos, se observa que apenas sobrepasan el mínimo de 60. El resultado 
se torna más preocupante si consideramos que dichos aspectos son enseñados a lo largo de las trayectorias escolares de los estudiantes hasta antes de ingresar a la universidad, por lo que esperaríamos que al incorporarse a una licenciatura debieran dominar los aspectos más elementales de la escritura. Sin embargo, vemos que no es así.

Se observa también que los resultados en la segunda parte del examen que tiene que ver con la comprensión lectora y la producción de textos, son todavía más críticos. En estos aspectos, los estudiantes obtuvieron puntajes menores a 60. Esta situación pone en evidencia que los docentes tenemos la urgente tarea de implementar estrategias didácticas que posibiliten el desarrollo de habilidades comunicativas en los estudiantes. Evidencia también que ésta no debe ser una tarea remedial, sino que debe instituirse y promoverse a través de acciones institucionales contextualizadas.

La importancia de evaluar el conocimiento y aplicación de aspectos normativos de la lengua en estudiantes universitarios, es que permite observar cómo expresan sus ideas, si lo hacen de manera coherente y eficiente, mientras que la relevancia de valorar aspectos de redacción y organización de ideas reside en que muestra su grado de comprensión en la lectura y sus habilidades para redactar textos propios. Problematizar estos aspectos en el contexto universitario nos obliga a la reflexión y a la búsqueda de estrategias alternativas que posibiliten su solución.

La lectura y la escritura en las distintas disciplinas son prácticas sociales institucionalizadas y contextualizadas, y el estudiante debe asumirlas para formar parte de la comunidad discursiva que le dará identidad. Son estas prácticas las que le permitirán realizar otras operaciones fundamentales de pensamiento como la inferencia, por ejemplo. Si el estudiante-lector no logra realizar el proceso inferencial al leer, no podrá tampoco establecer las relaciones de significado que se encuentran en los textos; no podrá interpretarlos, relacionarlos, clasificarlos o compararlos.

Como afirma Parodi (2005), una parte importante en el acceso al conocimiento especializado está mediado por el manejo eficiente de una competencia discursiva de corte disciplinar. La lectura y escritura académicas, así como la que se genera y circula en el ámbito 
profesional laboral, constituyen ejes fundamentales en la apropiación de los saberes, en la práctica cotidiana y en la integración definitiva como sujetos activos en esos grupos.

La comprensión de la lectura y la práctica de la escritura son temas de interés actual en todos los niveles educativos; ambas son habilidades necesarias para el aprendizaje significativo. Lo aprendido es significativo para los estudiantes cuando pueden integrar el nuevo conocimiento a sus esquemas cognitivos, cuando pueden relacionar ese conocimiento y aplicarlo a su realidad académica disciplinar. Una vez desarrolladas estas habilidades comunicativas, el estudiante aprenderá otras estrategias de apropiación de los contenidos, irá aprendiendo sobre su disciplina y podrá forjar su sentido de pertenencia a una comunidad discursiva.

El ámbito disciplinar al que pertenecen los estudiantes del Departamento de Contabilidad, así como el campo laboral al que se incorporarán una vez concluida su formación escolarizada, están inmersos en la cultura de la escritura normada; por ello es fundamental su aprendizaje. Nos corresponde a los docentes orientarlo y guiarlo a través de una enseñanza dialógica.

Finalmente, planteamos aquí algunas alternativas que pudieran apoyar el desarrollo de habilidades de lectoescritura en los estudiantes, y a la vez generar investigaciones posteriores.

\section{ALTERNATIVAS BÁSICAS DE SOLUCIÓN}

- Promover e impulsar a nivel institucional la realización de cursos y talleres de comprensión de lectura y de redacción para la planta docente. Es importante concientizarnos de que la lectura y la escritura son herramientas fundamentales en los procesos de aprendizaje y de enseñanza. Si enseñamos a los estudiantes a leer y a escribir en la universidad, ellos podrán apropiarse de los saberes propios de su disciplina y podrán también generarlos.

- Considerar la enseñanza de la lectura y la escritura como un eje transversal en los Planes de Estudios. Ello implica incluir más materias del área del lenguaje distribuidas entre los cinco ejes formativos que conforman a cada disciplina, lo cual permitiría relacionar y aplicar los contenidos.

Araoz, De la Varay García doi: https://doi.org/10.36799/el.v1i1.23 Volumen 1, Número 1, Año 2016, ISSN: 2448-5942 
- Brindar asesoría a los estudiantes durante las horas gabinete o como parte de las actividades del Programa Institucional de Tutorías (PIT), ya sea de manera presencial o virtual.

Promover y organizar proyectos estudiantiles de tutoría de pares a través de los programas de Prácticas Profesionales y de Servicio Social. Mediante estos proyectos, los estudiantes que poseen habilidades discursivas podrán apoyar a otros estudiantes que requieren desarrollarlas.

- Promover el trabajo colegiado interdisciplinar. Ello permitirá diseñar colaborativamente secuencias de trabajo con textos de estas áreas disciplinares y hará más factible que nuestros estudiantes puedan desempeñarse como mercadólogos-lectores, administradores-escritores o sociólogos-autores.

La importancia que actualmente tiene el tema de evaluar las habilidades discursivas de los estudiantes universitarios pone de manifiesto que es necesario un cambio de actitud del profesorado. Nos corresponde ahora ser mediadores y facilitadores del proceso de aprendizaje; sólo de esta manera podremos apoyar a nuestros estudiantes y brindarles las herramientas necesarias para que desarrollen y refuercen sus habilidades comunicativas.

Como se mencionó, la enseñanza de la lectura y la escritura en las diversas disciplinas no debe ser una práctica remedial ni un fin en sí mismo. Coincidimos con Miras, Solé y Castells cuando afirman que la lectura y la escritura son los medios que les posibilitarán su acceso a nuevos aprendizajes, así como "a la posibilidad de su uso autónomo e independiente como instrumento de disfrute y desarrollo personal” $(2012$, I 1$)$.

La mejor forma de aprender a leer y a escribir es la práctica constante, "leer y escribir forman parte del quehacer profesional/académico de los graduados que esperamos formar..." (Paula Carlino, 2012: 15).

\section{REFERENCIAS BIBLIOGRÁFICAS}

Araoz, De la Varay García doi: https://doi.org/10.36799/el.v1i1.23 Volumen 1, Número 1, Año 2016, ISSN: 2448-5942 
Álvarez Salas, Ana Laura; Fermín González Gaxiola y Minerva Eugenia Pardo Leal. Aspectos normativos de la lengua escrita. Hermosillo: Universidad de Sonora, STEUS, 2010.

Araoz Robles, María Edith, Patricia Guerrero de la Llata, Rosa Angélica Villaseñor Correa, María de los Ángeles Galindo Ruiz de Chávez y Ana Bertha De la Vara Estrada. Estrategias para aprender a aprender. Reconstrucción del conocimiento a partir de la lectoescritura. $2^{\mathrm{a}}$ ed. México: Pearson, Universidad de Sonora, 2010.

Calsamiglia Blancafort, Helena y Amparo Tusón Valls. Las cosas del decir. Manual de análisis del discurso. Barcelona: Ariel, 2007.

Carlino, Paula. Escribir, leer y aprender en la universidad. Una introducción a la alfabetización académica. Buenos Aires: Fondo de Cultura Económica, 2012.

Cassany, Daniel. Prácticas letradas contemporáneas. México: Ríos de tinta, 2008. Tras las líneas. Sobre la lectura contemporánea. Barcelona: Anagrama, 2006.

Castro Azuara, María Cristina (Coordinadora). Alfabetización académica y comunicación de saberes: la lectura y la escritura en la universidad. Tlaxcala: Universidad Autónoma de Tlaxcala, 2013.

Inzunza Inzunza, Vicente. Formulación y Evaluación de Proyectos de Inversión. Hermosillo: Universidad de Sonora, 2012.

Martínez, María Cristina. "Un procesamiento multinivel del texto escrito. ¿Un giro discursivo en los estudios sobre la comprensión de textos?” En Quintana, Hilda. (Comp.) Entre libros y papeles. Memorias del Primer Simposio de la Cátedra UNESCO para el Mejoramiento de la Lectura y la Escritura en América Latina, (23-45). Colombia: Universidad Interamericana de Puerto Rico, Cátedra UNESCO. 2004.

Miras, Mariana, Isabel Solé y Núria Castells. "Evaluación en el área de la lengua: pruebas escritas y opiniones de los profesores". Lectura y vida. Revista Latinoamericana de Lectura. Año 21, no. 2, 2000. Recuperado el 18 enero de 2013 de http://www.lecturayvida.fahce.unlp.edu.ar/numeros/a21n2/21_02_Miras.pdf

Munch Galindo, Lourdes. Métodos y técnicas de investigación. México: Trillas, 2009. 
Parodi, Giovanni. "La comprensión del discurso especializado escrito en ámbitos técnicoprofesionales: ¿Aprendiendo a partir del texto?” Revista signos, 38.58. (2005): 221-267. Consultado el 11 de septiembre de 2014. http://dx.doi.org/10.4067/S071809342005000200005 